\title{
Digital speckle pattern interferometry applied to a surface roughness study
}

\author{
Mario Lehman* \\ Centro de Investigaciones Opticas (ClOp) \\ C. C. $124-1900$ \\ La Plata, Buenos Aires, Argentina \\ Juan A. Pomarico \\ Roberto D. Torroba \\ Centro de Investigaciones Opticas (ClOp) \\ C. C. $124-1900$ \\ La Plata, Buenos Aires, Argentina \\ and \\ Universidad Nacional de La Plata \\ Facultad de Ingeniería \\ La Plata, Buenos Aires, Argentina
}

\begin{abstract}
Surface roughness determination is of great interest for many applications. Several methods can be found in the literature, but most of them rely on indirect evaluation of the information, such as photographic techniques. We propose a method to measure surface roughness that takes advantage of digital speckle pattern interferometry for obtaining the data, and of digital image processing for evaluating it. After defining the problem, a theoretical description is presented, and finally it is compared with experimental results, showing good agreement.
\end{abstract}

Subject terms: digital speckle pattern interferometry; speckle statistics; roughness measurement; interferometry.

Optical Engineering 34(4), 1148-1152 (April 1995).

\section{Introduction}

Since the fundamental work of Beckmann et al. ${ }^{1}$ dealing with the scattering of electromagnetic waves from rough surfaces, their results have been extensively used to obtain information about surface roughness from the scattered light in the farfield region.

The correlation fringes of two speckle patterns, obtained from a rough surface under different angles of illumination, bear information about the roughness. Several optical roughness measurements techniques involve the use of a doubleexposure specklegram method. The basic approach is to produce a shift in the incidence angle of the illumination beam between exposures, in order to produce, after a Fourier transformation, a set of interference fringes whose visibility depends on the rms roughness of the scattering surface..$^{2,3}$ This technique is known as angular speckle correlation (ASC).

Tribillon ${ }^{4}$ showed that the degree of correlation between speckle patterns generated by two different wavelengths depends strongly on the surface roughness. The method based on this effect is known as spectral speckle correlation (SSC). ${ }^{5}$

All of the above-mentioned techniques suffer from the disadvantages of a photographic process, besides introducing a second step in the use of an optical Fourier transform. There also exist statistical methods that derive roughness information from the degree of decorrelation of imaged speckle patterns as a function of the wavelength, ${ }^{6}$ where electronic detection and processing replaces the conventional photographic record. Electronic correlation of speckle patterns is currently accomplished by a technique called digital speckle

*Current affiliation: Universidad de Bahía Blanca, Buenos Aires, Argentina.

Paper 09074 received July 12, 1994; revised manuscript received Nov. 1, 1994; accepted for publication Nov. 1, 1994.

(c) 1995 Society of Photo-Optical Instrumentation Engineers. 0091-3286/95/\$6.00. pattern interferometry (DSPI), normally used in various metrological applications. ${ }^{7}=9$

In DSPI a fringe pattern is obtained using video processing by storing an initial reference image of the surface in a frame grabber and then subtracting it from the one being acquired, displaying the modulus of the result on a TV monitor. Fringes, similar to those obtained in holographic interferometry, then appear when a phase change is introduced between the reference image and the subsequent ones. As noted by Wykes, ${ }^{10}$ the rms difference of the speckle intensities from different wavelengths is directly related to the degree of speckle correlation.

In this work we apply the DSPI technique on a singlewavelength basis, combined with the ASC method, to show that the visibility of the correlation fringes in this case also depends on the roughness of the object surface. A theoretical approach to the proposed technique is also given.

\section{Theoretical Description}

Let us suppose the situation sketched in Fig. 1. A plane wave $\Sigma$ illuminates a rough surface $S$ at an angle of incidence $\theta$ with respect to the normal to the surface. In our case, the surface is considered to be formed from a set of $n$ identical scattering elements of random height following a normal distribution of standard deviation $\sigma$ and zero mean. A CCD camera images the surface on the detector array. A separate reference beam is added to the first one via the beamsplitter BS. Since the DSPI detects a quantity proportional to $\left|\left\langle I_{1}-I_{2}\right)^{2}\right\rangle^{1 / 2} \mid$, we examine how this quantity varies with a tilt of the beam illuminating the surface.

To do that we assume

$A_{1}=A_{0} \sum_{j=1}^{n} \exp \left\{\frac{2 \pi i}{\lambda}\left[Z_{j}(1+\cos \theta)+\phi_{0}\right]\right\}+A_{R} \exp \left(i \phi_{R}\right)$ 

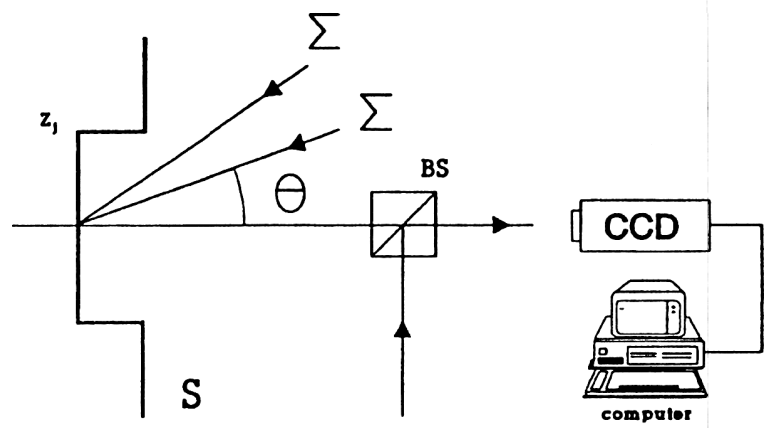

Fig. 1 Schematic representation of two plane waves, $\Sigma$ and $\Sigma^{\prime \prime}$, itluminating a rough surface at two different angles, $\theta$ and $\theta+\Delta \theta$.

to be the field amplitude detected by the $\mathrm{CCD}$ array when the surface is illuminated at an angle $\theta$. In this equation the first term denotes the speckle pattern amplitude field, where $A_{0}$ is the light amplitude scattered by one element, $Z_{j}$ is the height of the $j$ th scattering element, and $\phi_{0}$ allows for the constant phase of the incident light. In the second term, $A_{R}$ is the amplitude of the reference beam and $\phi_{R}$ its phase. In the following, all calculations are assumed to be made at the same point $(x, y)$.

Similarly, if the illumination direction is changed by an amount $\Delta \theta$, the new field amplitude can be described by

$$
\begin{aligned}
A_{2}= & A_{0} \sum_{j=1}^{n} \exp \left(\frac{2 \pi i}{\lambda}\left\{Z_{j}[1+\cos (\theta+\Delta \theta)]+\phi_{0}\right\}\right) \\
& +A_{R} \exp \left(i \phi_{R}\right) .
\end{aligned}
$$

Equations (1) and (2) can be rewritten as the following more compact expressions:

$A_{1}=A_{0} e^{i \phi} \sum_{j=1}^{n} e^{i \alpha Z_{j}}+A_{R} e^{i \beta}$

and

$A_{2}=A_{0} e^{i \phi} \sum_{j=1}^{n} e^{i \gamma Z_{j}}+A_{R} e^{i \beta}$,

where $e^{i \phi}$ is the common phase term $\exp \left[i 2 \pi \phi_{0} / \lambda\right], A_{R}$ is the amplitude of the reference beam, and $\alpha$ and $\gamma$ are abbreviations for $(2 \pi / \lambda)(1+\cos \theta)$ and $(2 \pi / \lambda)[1+\cos (\theta+\Delta \theta)]$, respectively.

To obtain $\left\langle\left(I_{1}-I_{2}\right)^{2}\right\rangle$ we need to calculate $\left\langle I_{1}^{2}\right\rangle,\left\langle I_{2}^{2}\right\rangle$ and $\left\langle I_{1} I_{2}\right\rangle$, since

$\left\langle\left(I_{1}-I_{2}\right)^{2}\right\rangle=\left\langle I_{1}^{2}\right\rangle+\left\langle I_{2}^{2}\right\rangle-2\left\langle I_{1} I_{2}\right\rangle$.

Using Eqs. (3) and (4) we obtain

$$
\begin{aligned}
I_{1}= & A_{1} A_{1}^{*} \\
= & A_{0}^{2} \sum_{j=1}^{n} e^{i \alpha Z_{j}} \sum_{k=1}^{n} e^{-i \alpha Z_{k}}+A_{R}^{2}+A_{0} B e^{i(\phi-\beta)} \sum_{j=1}^{n} e^{i \alpha Z_{j}} \\
& +A_{0} A_{R} e^{-i(\phi-\beta)} \sum_{j=1}^{n} e^{-i \alpha Z_{j}}
\end{aligned}
$$

and

$$
\begin{aligned}
I_{2}= & A_{2} A_{2}^{*} \\
= & A_{0}^{2} \sum_{j=1}^{n} e^{i \gamma Z_{j}} \sum_{k=1}^{n} e^{-i \gamma Z_{k}}+A_{R}^{2}+A_{0} A_{R} e^{i(\phi-\beta)} \sum_{j=1}^{n} e^{i \gamma Z_{j}} \\
& +A_{0} A_{R} e^{-i(\phi-\beta)} \sum_{j=1}^{n} e^{-i \gamma Z_{j}} .
\end{aligned}
$$

Equations (6) and (7) can be reduced to the following expressions:

$$
\begin{aligned}
I_{1}= & A_{0}^{2}\left(n+2 \sum_{\substack{j=1 \\
j<k}}^{n-1} \cos \alpha\left(Z_{j}-Z_{k}\right)\right)+A_{R}^{2} \\
& +2 A_{0} A_{R} \sum_{j=1}^{n} \cos \left(\alpha Z_{j}+\phi-\beta\right), \\
I_{2}= & A_{0}^{2}\left(n+2 \sum_{j=1}^{n-1} \cos \gamma\left(Z_{j}-Z_{k}\right)\right)+A_{R}^{2} \\
& +2 A_{0} A_{R} \sum_{j=1}^{n} \cos \left(\gamma Z_{j}+\phi-\beta\right) .
\end{aligned}
$$

Squaring Eq. (8) yields

$$
\begin{aligned}
I_{1}^{2}= & A_{0}^{4} n^{2}+4 n A_{0}^{4} \sum_{j<k} \cos \alpha\left(Z_{j}-Z_{k}\right) \\
& +4 A_{0}^{4} \sum_{j<k} \cos ^{2} \alpha\left(Z_{j}-Z_{k}\right)+A_{R}^{4} \\
& +8 A_{0}^{4} \sum_{\substack{j<k \\
j \neq m}} \cos \alpha\left(Z_{j}-Z_{k}\right) \cos \alpha\left(Z_{l}-Z_{m}\right) \\
& +4 A_{0}^{2} A_{R}^{2} \sum_{j=1}^{n} \cos ^{2}\left(\alpha Z_{j}+\phi-\beta\right) \\
& +8 A_{0}^{2} A_{R}^{2} \sum_{j<k} \cos \left(\alpha Z_{j}+\phi-\beta\right) \cos \left(\alpha Z_{k}+\phi-\beta\right) \\
& +2 A_{0}^{2} A_{R}^{2}\left(n+2 \sum_{j<k} \cos \alpha\left(Z_{j}-Z_{k}\right)\right) \\
& +4 A_{0}^{3} A_{R} n \sum_{j=1}^{n} \cos \left(\alpha Z_{j}+\phi-\beta\right) \\
& +8 A_{0}^{3} A_{R} \sum_{j<k} \cos \alpha\left(Z_{j}-Z_{k}\right) \cos \left(\alpha Z_{l}+\phi-\beta\right) \\
& +4 A_{0} A_{R}^{3} \sum_{j=1}^{n} \cos \left(\alpha Z_{j}+\phi-\beta\right)
\end{aligned}
$$


from which we obtain, taking into account ${ }^{11}$ that the mean value of the cosine function evaluates to zero when the phase is uniformly distributed in the interval $[-\pi, \pi]$,

$\left\langle I_{1}^{2}\right\rangle=A_{0}^{4} n^{2}+4 A_{0}^{4} \frac{n^{2}-n}{4}+A_{R}^{4}+4 A_{0}^{2} A_{R}^{2} \frac{n}{2}+2 A_{0}^{2} A_{R}^{2} n$.

Clearly, since this last expression for $\left\langle I_{1}^{2}\right\rangle$ does not depend on either $\alpha$ or on $\gamma$, the same result holds for $\left\langle I_{2}^{2}\right\rangle$.

To obtain the third term in Eq. (5) we need now to calculate the mean value of the product $I_{1} I_{2}$ :

$$
\begin{aligned}
I_{1} I_{2}= & A_{0}^{4}\left[n^{2}+2 n\left(\sum_{j<k} \cos \alpha\left(Z_{j}-Z_{k}\right)+\sum_{j<k} \cos \gamma\left(Z_{j}-Z_{k}\right)\right)\right. \\
& \left.+4 \sum_{j<k} \cos \alpha\left(Z_{j}-Z_{k}\right) \sum_{j<k} \cos \gamma\left(Z_{j}-Z_{k}\right)\right] \\
& +A_{R}^{4}+4 A_{0}^{2} A_{R}^{2}\left(\sum_{j=1}^{n} \cos \left(\alpha Z_{j}+\phi-\beta\right) \sum_{j=1}^{n}\right. \\
& \left.\left.\times \cos \left(\gamma Z_{j}+\phi-\beta\right)\right)\right]+A_{0}^{2} A_{R}^{2}\left[2 n+2\left(\sum_{j<k}\right.\right. \\
& \left.\left.\times \cos \alpha\left(Z_{j}-Z_{k}\right)+\sum_{j<k} \cos \gamma\left(Z_{j}-Z_{k}\right)\right)\right]+2 A_{0}^{3} A_{R} \\
& \times\left[\left(n+2 \sum_{j<k} \cos \alpha\left(Z_{j}-Z_{k}\right)\right) \sum_{j=1}^{n} \cos \left(\gamma Z_{j}+\phi-\beta\right)\right. \\
& \left.+\left(n+2 \sum_{j<k} \cos \gamma\left(Z_{j}-Z_{k}\right)\right) \sum_{j=1}^{n} \cos \left(\alpha Z_{j}+\phi-\beta\right)\right] \\
& +2 A_{0} A_{R}^{3}\left(\sum_{j=1}^{n} \cos \left(\alpha Z_{j}+\phi-\beta\right)+\sum_{j=1}^{n}\right. \\
& \left.\times \cos \left(\gamma Z_{j}+\phi-\beta\right)\right) .
\end{aligned}
$$

Taking into account that

$$
\begin{aligned}
\cos \alpha\left(Z_{j}-Z_{k}\right) \cos \gamma\left(Z_{j}-Z_{k}\right)= & \frac{1}{2}\left\{\cos \left[(\alpha+\gamma)\left(Z_{j}-Z_{k}\right)\right]\right. \\
& \left.+\cos \left[(\alpha-\gamma)\left(Z_{j}-Z_{k}\right)\right]\right\},
\end{aligned}
$$

the mean value $\left\langle\cos \alpha\left(Z_{j}-Z_{k}\right) \cos \gamma\left(Z_{j}-Z_{k}\right)\right\rangle$ evaluates to

$\frac{1}{2}\left\langle\cos (\alpha-\gamma)\left(Z_{j}-Z_{k}\right)\right\rangle$.

Taking into account that we assumed a normal distribution with standard deviation $\sigma$ and zero mean, this last equation can be expressed as

$\frac{1}{2}\left\langle\exp \left[i(\alpha-\gamma)\left(Z_{j}-Z_{k}\right)\right]\right\rangle$.

This ensemble average evaluates to

$\frac{1}{2} \exp \left(-\frac{1}{2}(\alpha-\gamma)^{2}\left\langle\left(Z_{j}-Z_{k}\right)^{2}\right\rangle\right)$ and $\left\langle\left(Z_{j}-Z_{k}\right)^{2}\right\rangle=2 \sigma^{2}\left(1-r_{j k}\right)$, where $r_{j k}$ is the normalized surface correlation. Since from Eq. (12) we have $j \neq k$, and if the individual steps $Z_{j}$ are uncorrelated with each other, then Eq. (13) reduces to

$\frac{1}{2} \exp \left[-(\alpha-\gamma)^{2} \sigma^{2}\right]$.

With this result, the final expression for $\left\langle I_{1} I_{2}\right\rangle$ becomes

$$
\begin{aligned}
\left\langle I_{1} I_{2}\right\rangle= & A_{0}^{4}\left(n^{2}+2 \frac{n^{2}-n}{2} \exp \left(-\xi^{2} \sigma^{2}\right)\right) \\
& +A_{R}^{4}+2 n A_{0}^{2} A_{R}^{2} \exp \left(-\frac{\xi^{2} \sigma^{2}}{2}\right)+2 n A_{0}^{2} A_{R}^{2},
\end{aligned}
$$

where $\xi \equiv \alpha-\gamma \equiv(2 \pi / \lambda)[\cos \theta-\cos (\theta+\Delta \theta)] \approx(2 \pi / \lambda) \sin \theta$ $\Delta \theta$. From Eqs. (11) and (16) the following expression for $\left\langle\left(I_{1}-I_{2}\right)^{2}\right\rangle$ can be obtained:

$$
\begin{aligned}
\left\langle\left(I_{1}-I_{2}\right)^{2}\right\rangle= & 2 n A_{0}^{2}\left\{(n-1) A_{0}^{2}\left[1-\exp \left(-\xi^{2} \sigma^{2}\right)\right]\right. \\
& \left.+2 A_{R}^{2}\left[1-\exp \left(-\frac{\xi^{2} \sigma^{2}}{2}\right)\right]\right\} .
\end{aligned}
$$

The number of scatterers producing the speckle field, $n$, is very large, and thus $n-1 \approx n$. In addition, we suppose that $n A_{0}^{2}=A_{S}^{2}$ represents the total light intensity emanating from the surface and $A_{R}$ can be chosen to be equal to $A_{S}$. With these assumptions, the final expression for $\left\langle\left(I_{1}-I_{2}\right)^{2}\right\rangle$ results:

$$
\left\langle\left(I_{1}-I_{2}\right)^{2}\right\rangle=2 A_{S}^{4}\left[3-\exp \left(-\xi^{2} \sigma^{2}\right)-2 \exp \left(-\frac{\xi^{2} \sigma^{2}}{2}\right)\right] .
$$

We are primarily concerned with the determination of the local visibility $V_{L}$ and its variation with respect to $\Delta \theta$. By using the substitution $\left\langle\left(I_{1}-I_{2}\right)^{2}\right\rangle \equiv\left\langle I^{2}\right\rangle$, the local visibility is defined as

$$
V_{L}=\frac{\left\langle I^{2}\right\rangle_{\max }^{1 / 2}-\left\langle I^{2}\right\rangle_{\min }^{1 / 2}}{\left\langle I^{2}\right\rangle_{\max }^{1 / 2}+\left\langle I^{2}\right\rangle_{\min }^{1 / 2}}
$$

In practice, it is equivalent to measure the global visibility through the fringe pattern that arises because of the tilt $\Delta \theta$ of the illuminating beam, defined by the equation

$V=\frac{I_{\max }-I_{\min }}{I_{\max }+I_{\min }}$,

where $I_{\max }$ and $I_{\min }$ are the maximum and minimum intensity values present in the fringe pattern, respectively.

For the case of $\Delta \theta \equiv 0$, we have $\left\langle\left(I_{1}-I_{2}\right)^{2}\right\rangle^{1 / 2}=0$ (no fringes are present), and Eq. (19) becomes undefined. The corresponding visibility can be taken as one, as for all other cases, having $\Delta \theta \neq 0$, lower values are obtained.

Figure 2 shows the variation of the local visibility defined in Eq. (19) versus the rotation $\Delta \theta$ for $\sigma=6.3 \mu \mathrm{m}$. 


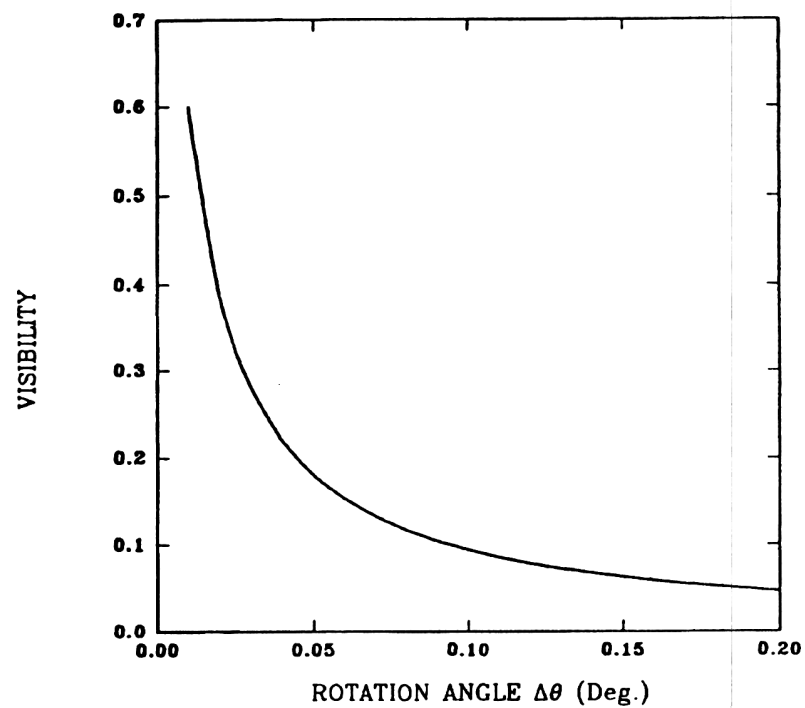

Fig. 2 Theoretical variation of the visibility as a function of the change in the illumination direction, $\Delta \theta$.

\section{Experimental Results}

The experimental arrangement of Fig. 3 was used to reproduce the conditions used in the theoretical approach. A $10-\mathrm{mW} \mathrm{HeNe}$ laser beam was divided by the beamsplitter BS1. One of the beams obtained in this way was expanded, collimated, and used to illuminate the rough surface under study at an angle $\theta$ with its normal. The second beam was overlapped with the image of the surface, which is a speckle pattern, at the detector array of the CCD camera by using the beamsplitter BS2. It is clear that the optical system does not resolve the individual scatterers. The video signal from the camera is sent to the frame grabber for further processing.

At the frame grabber the following operation, which can be accomplished at near-real-time rates, is performed: a first image of the surface is acquired and stored as a reference,

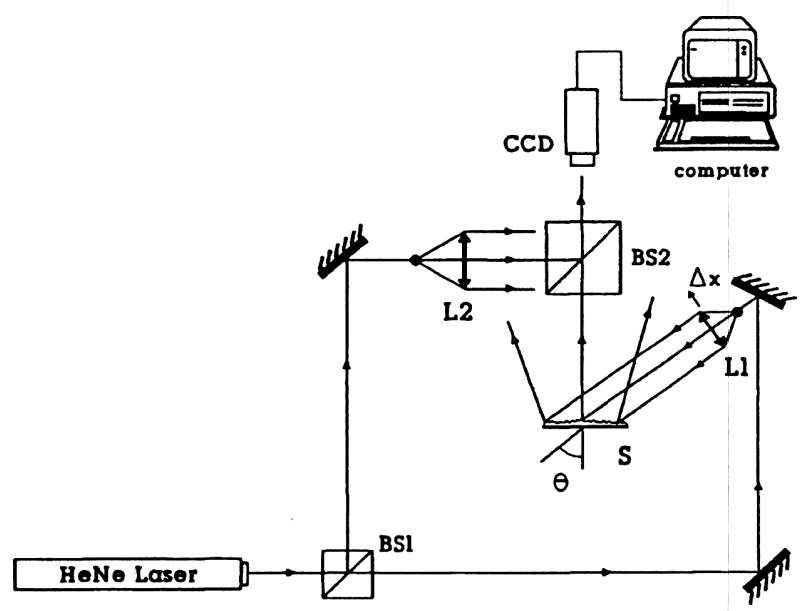

Fig. 3 Experimental DSPI setup for obtaining speckle correlation fringes and measuring the angular variation $\Delta \theta$.

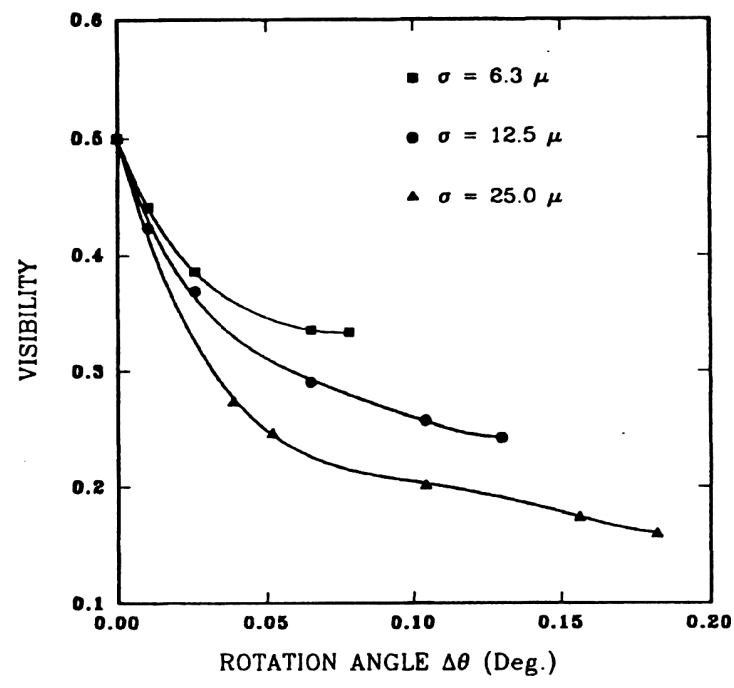

Fig. 4 Visibility of the fringe pattern as a function of $\Delta \theta$ for three different values of the surface roughness. Solid curves represent the best fit to the experimental data.

the subsequent images coming from the camera are subtracted from the first one, and the result is then displayed, in modulus, on a TV monitor. If an image is obtained under the same illumination conditions as the reference, then no differences are present between them, and the TV monitor appears dark. On the other hand, when the beam illuminating the surface is rotated by an angle $\Delta \theta$, a linear phase change occurs, altering the speckle pattern generated by the surface. At points where this phase change equals $2 m \pi$, with $m$ an integer, the speckle pattern reproduces itself and the TV monitor shows a dark spot. On the contrary, for phase changes other than $2 m \pi$, the subtraction gives a nonzero value and the monitor shows a spot whose gray level varies accordingly to the phase change. Thus the monitor shows a set of fringes immersed in speckle noise and following the zones of constant phase change. Both the spatial frequency and the visibility of these fringes depends on the rotation $\Delta \theta$ of the beam illuminating the surface, as demonstrated above.

In our experiment, the rotation $\Delta \theta$ was obtained by a lateral shift $\Delta x$ of the collimating lens $L 1$, which was mounted on a translator driven by a stepper motor capable of a minimum displacement of $2 \mu \mathrm{m}$. Lens $\mathrm{L} 1$ was chosen to have a focal length of $22 \mathrm{~cm}$. Thus, the rotation $\Delta \theta$ can be obtained from the simple equation

$\tan \Delta \theta=\frac{\Delta x}{f} \approx \Delta \theta$,

with $\Delta x$ varying from several microns to a few millimeters.

Although this produces not only a rotation but also a displacement of the object beam, using plane waves ensures that all phase changes are only a consequence of the rotation, provided that the whole field of view of the camera (which can always be chosen smaller than the illuminated area) remains unchanged.

The curves plotted in Fig. 4 (fringe visibility as a function of $\Delta \theta$ ) were obtained for three different values of the surface roughness, $\sigma \equiv 6.3,12.5$, and $25 \mu \mathrm{m}$, and for $\theta \equiv 19 \mathrm{deg}$, 


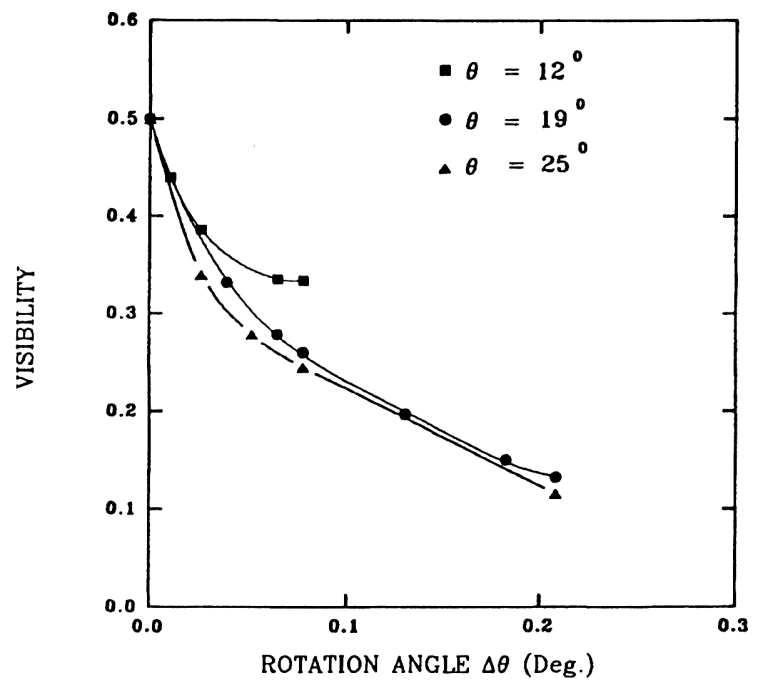

Fig. 5 Best-fit curves for the visibility of the fringe pattern as a function of $\Delta \theta$ for three different values of the initial illumination angle $\theta$ and for a fixed value of the surface roughness, $\sigma=6.3 \mu \mathrm{m}$.

using standard surface roughness test plates. Clearly the visibility drops faster as the roughness increases, as can be expected from Eq. (18). Visibility measurements were carried out using an indirect digital method, ${ }^{12}$ where a peak ratio at a Fourier plane is measured, instead of direct fringe visibility analysis. In this way, speckle noise is reduced and the error in the visibility remains around $1.4 \%$.

For a given roughness, if the incidence angle $\theta$ of the beam illuminating the surface is changed, the maximum value of $\Delta \theta$ for which a variation in the fringes' visibility can be detected also changes, as illustrated in Fig. 5 for $\sigma \equiv 6.3 \mu \mathrm{m}$ and $\theta=12,19$, and $25 \mathrm{deg}$. The sensitivity of the method (which is limited by the fringe density) can thus be controlled by setting the proper value of $\theta$.

For both, Figs. 4 and 5, solid curves represent the best fit to the experimental values.

\section{Conclusions}

A method for surface roughness determination using digital speckle pattern interferometry has been described theoretically and tested experimentally, showing good agreement. The experimental setup requires an image processor but not sophisticated optical components, making the method suitable, for example, for industrial applications. In addition, the whole process can be accomplished digitally, thus reducing the time needed for a measurement. The range of operation can be controlled by adjusting the parameters.

\section{Acknowledgments}

This work was supported by Fundación Antorchas (Argentina), the Alexander Von Humboldt Foundation (Germany), and CONICET PID Nr.:3974/912 (Argentina). We also thank B. Ruiz for her valuable help with the mathematics involved in this work.

\section{References}

1. P. Beckmann and A. Spizzichino, The Scattering of Electromagnetic Waves from Rough Surfaces, Pergamon, Oxford (1963).

2. D. Léger, E. Mathieu, and J. C. Perrin, "Optical surface roughness determination using speckle correlation technique," Appl. Opt. 14(4), 872-877 (1975)

3. D. Léger and J. C. Perrin, " Real-time measurement of surface roughness by correlation of speckle patterns," J. Opt. Soc. Am. 66(11), 1210-1217 (1976).

4. G. Tribillon, "Correlation entre deux speckle obtenus avec deux longueurs d'onde-application à la mesure de la rugosité moyenne," Opt. Commun. 11(2), 172-174 (1974).

5. B. Ruffling, "Application of speckle-correlation methods to surfaceroughness measurement: a theoretical study," J. Opt. Soc. Am. A 3(8), 1297-1304 (1986)

6. D. J. Schertler and N. George, "Roughness determination by specklewavelength decorrelation," Opt. Lett. 18(5), 391-393 (1993).

7. C. Joenathan and R. Torroba, "Simple electronic speckle-shearingpattern interferometer," Opt. Lett. 15(20), 1159-1161 (1990).

8. N. Bolognini, H. Rabal, and R. Torroba, "Single-beam digital holographic contouring," Appl. Opt. 31(8), 1009-1011 (1992).

9. J. Pomarico, R. Arizaga, H. Rabal, and R. Torroba, "Digital holographic contouring," Opt. Commun. 98(4), 257-260 (1993).

10. C. Wykes, "De-correlation effects in speckle-pattern interferometry. 1. Wavelength change dependent de-correlation with application to contouring and surface roughness measurement," Opt. Acta 24(5), 517-532 (1977).

11. J. W. Goodman, "Statistical properties of laser speckle patterns," Chapter 1 in Laser Speckle and Related Phenomena, J. C. Dainty, Ed. pp. 9-26, Springer-Verlag, Berlin (1975).

12. J.Pomarico and R. Torroba, "Digital visibility measurements by Fourier analysis," Optik 95(4), 152-154 (1994).

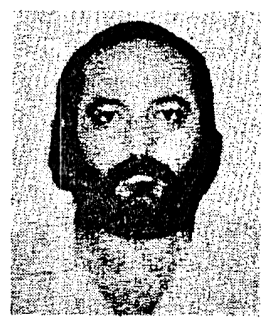

Mario Lehman received his MS degree from the Universidad del Centro de la Provincia de Buenos Aires, Tandil, Argentina, and teaches physics at the Universidad Nacional del Sur, Bahia Blanca. He is working at the Optical Research Center (CIOp) toward his PhD in the field of digital speckle pattern interferometry and optical metrology.

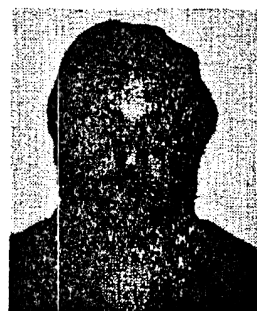

Juan A. Pomarico received his MS and PhD degrees from the University of San Luis, Argentina, in 1985 and 1993, respectively. He teaches physics in the Faculty of Engineering of the University of La Plata and is a researcher at the Optical Research Center (ClOp), La Plata. He has been a research fellow of the German Academic Exchange Service (DAAD) at Stuttgart University (Germany), and of the International Center for Theoretical Physics (ICTP), Italy. His research interests include TV holography and optical metrology.

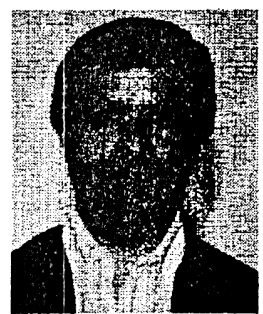

Roberto D. Torroba received his MS and PhD degrees in physics from the University of La Plata, Argentina, in 1977 and 1982, respectively. He is currently a professor at the Faculty of Engineering of the University of La Plata. Since 1977 he has been in the Optics Group at the Optical Research Center (ClOp), La Plata. He is a member of the Argentinian Research Council (CONICET) and an associate researcher at the International Center for Science and High Technology (ICS), Trieste, Italy. He has been a fellow of the Alexander von Humboldt Stiftung (Germany), the German Academic Exchange Service (DAAD), the Organization of American States, and the Valencian Community (Spain). His current research interests include TV holography and optical metrology. 